\title{
LAS SALINAS CONTINENTALES DE ANDALUCÍA: RECURSO ECO-CULTURAL CON POTENCIALIDAD DIDÁCTICA Y TURÍSTICA
}

\author{
Rafael Vega-Pozuelo \\ Martín Torres-Márquez \\ José Naranjo-Ramírez \\ Universidad de Córdoba
}

\section{RESUMEN}

El objetivo de esta aportación es la descripción de los vínculos existentes y potenciales entre turismo, educación, territorio y el patrimonio salinero continental, tomando como caso de estudio una zona concreta de Andalucía (España). Pretendemos, desde la perspectiva de la geografía ambiental, ofrecer las potencialidades turísticas y educativas de unos humedales culturales concretos: las salinas de interior. Estas ofrecen unas posibilidades únicas desde la diversidad ambiental y cultural de este espacio como destino de flujos turísticos y/o educativos.

Palabras clave: Patrimonio; salina continental; Andalucía; turismo.

Continental cultural salt mines in Andalusia: eco-cultural resource with didactic and tourist potentiality

\begin{abstract}
To describe the existing and potential links between tourism, education, territory and the continental saltland heritage is the objective of this contribution is, taking as a case study a specific area of Andalusia (Spain). We aim, from the perspective of environmental geography, to offer the tourist and educational potential of some specific cultural wetlands: the salt flats of the interior. These offer unique possibilities from the environmental and cultural diversity of this space as a destination of tourist and / or educational flows.
\end{abstract}

Keywords: Heritage; Continental saline; Andalusia; Tourism.

Fecha de recepción: 1 de septiembre de 2017.

Fecha de aceptación: 20 de marzo de 2018.

Departamento de Geografía y Ciencias del Territorio. Universidad de Córdoba. Plaza Cardenal Salazar, 3. 14071 CÓRDOBA (España).E-mail: a72vepor@uco.es, martin.torres@uco.es, ch1naraj@hotmail.com 


\section{PREMISA}

El presente trabajo forma parte de un proyecto más amplio en el que, con finalidad doctoral de uno de los autores, se aspira a estudiar y caracterizar el conjunto de láminas de agua (charcas, lagunas y salinas) que de forma frecuente se localizan en la zona central de Andalucía. Dicho proyecto está planteado en el espacio de la cuenca del Guadalquivir comprendido entre dos de sus afluentes meridionales: el Guadalbullón, por el este, y el Genil, por el oeste, afectando a 89 términos municipales pertenecientes a las provincias de Jaén, Córdoba, Granada y Sevilla.

Se pretende, así mismo, aportar la suficiente información para comprender las bases científicas más relevantes de un patrimonio industrial en patente proceso de extinción como es el caso de las salinas continentales y que pudiera servir como importante recurso en propuestas de reutilización turística, cultural y didáctica, así como para la revitalización económica de esta zona del centro de Andalucía, pues posee un patrimonio arquitectónico, paisajístico y natural de amplias posibilidades, aunque en proceso de abandono y desaparición.

Este propósito no resulta fácil por la propia complejidad del tema de estudio y por la escasez de referencias metodológicas y conceptuales desde el ámbito de la geografía humana y ambiental.

\section{MARCO TERRITORIAL}

\section{Figura 1}

\section{IDENTIFICACIÓN DEL ÁREA DE ESTUDIO EN EL CONTEXTO DE LAS CUENCAS HIDROGRÁFICAS QUE DISCURREN POR EL TERRITORIO DE ANDALUCÍA}

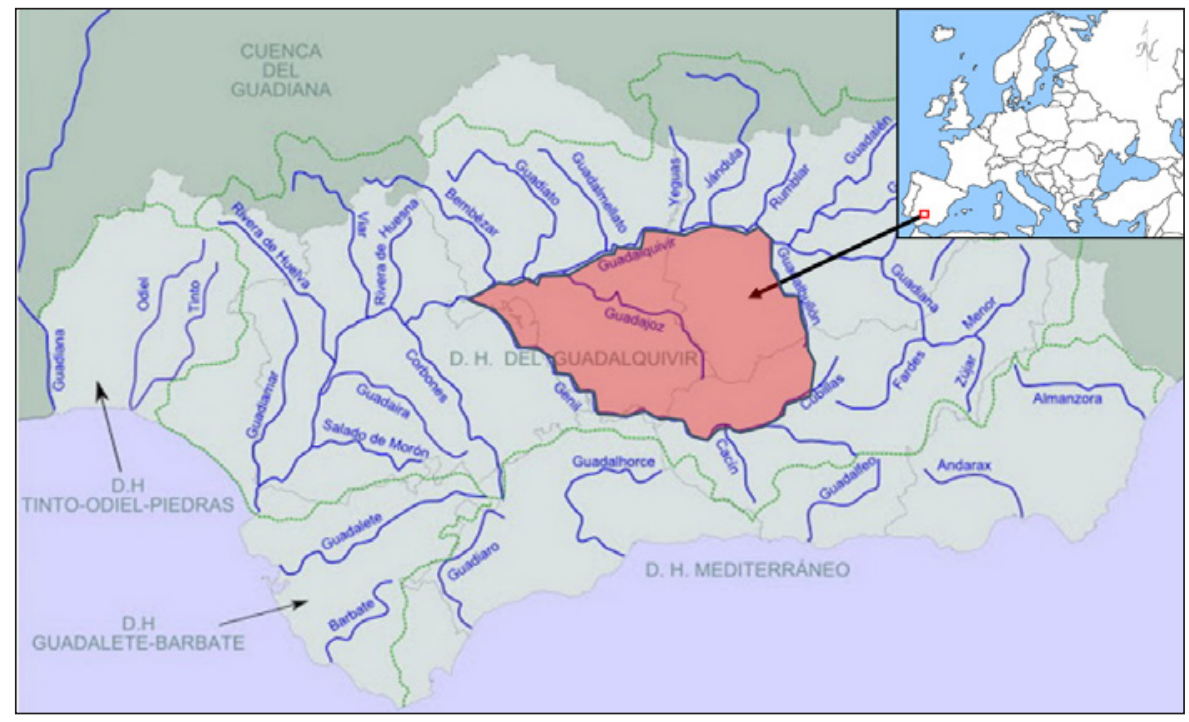

Fuente: Plan Hidrológico del Guadalquivir 2009-2015. 
Para el estudio de propuestas de itinerarios turísticos y educativos relacionados con la actividad salinera continental en la cuenca media del Guadalquivir, encontramos la necesidad de profundizar en la caracterización de la realidad territorial de la zona de estudio: el área dispuesta entre los ríos Guadalbullón y Genil. De esta forma, podremos avanzar a la vez que considerar la tipología de este espacio turístico y educativo, entendido como categoría de análisis y como herramienta para su estudio (López Palomeque, 1993).

En el contexto del centro de Andalucía, la zona que estudiamos se integra en el proceso de sedimentación miocena, y es el resultado del relleno del Golfo Bético tras el plegamiento Alpino, con la progresiva elevación de los fondos marinos por colmatación. Lejos de lo que suele pensarse la zona de estudio presenta una apreciable diversidad subcomarcal. Desde el Valle estricto del Guadalquivir (construido sobre las terrazas del río), a la Campiña Baja o "Campiña de Córdoba", pasando por la concreción (en el seno de esta Campiña Baja) de los "municipios carolinos" (con su glacis Villafranquiense); la Campiña Alta -a la que nos vamos a referir- viene a cerrar este gran espacio en su contacto final con las primeras estribaciones subbéticas (López Ontiveros, 2005; Naranjo Ramírez, 2013).

\subsection{Los rasgos físicos básicos}

Figura 2

SALINAS DE TEJERO, EN SANTIAGO DE CALATRAVA, JAÉN

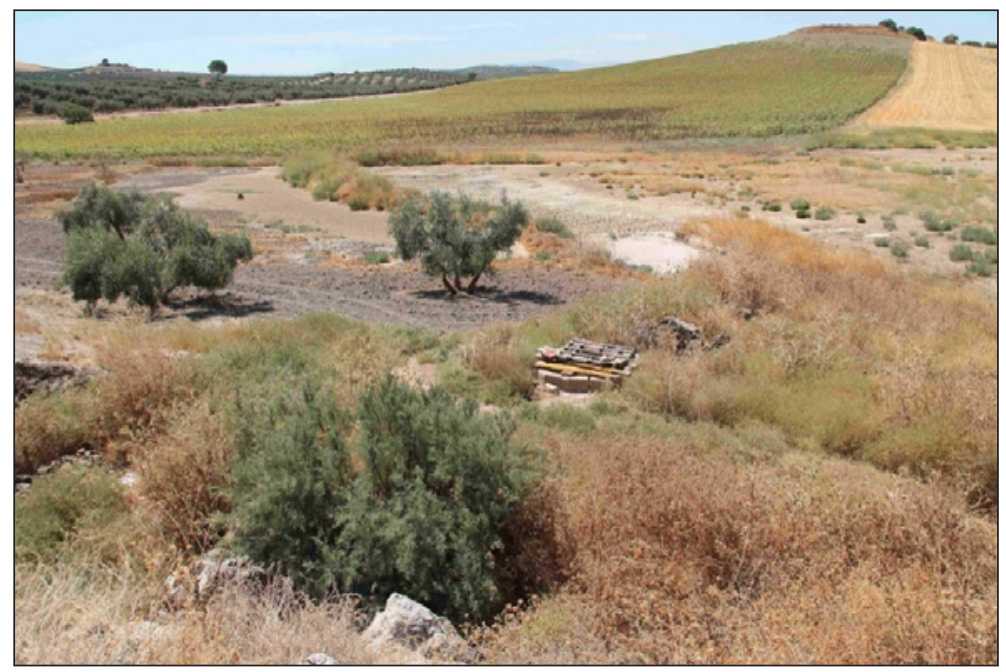

Autor: Rafael Vega-Pozuelo.

Dentro de nuestra zona de estudio destacamos la topografía campiñesa, que presenta un relieve de formas suaves y alomadas, un conjunto de cerros y colinas de perfil redondeado, distribuidas anárquicamente y sin líneas de relieve perceptibles. Son resultado de la acción erosiva de ríos y arroyos que, al incidir sobre los blandos terrenos terciarios, 
acaban fragmentando los paquetes sedimentarios superiores y, a sus expensas, crean nuevas subunidades, nuevas lomas o colinas. Estas líneas de escorrentía muchas veces ni siquiera tienen carácter permanente, pero ello no les resta eficacia erosiva en virtud de la propia blandura de los materiales y de la torrencialidad estacional de las aguas (Dantín, 1940; Recio, 1989; Díaz del Olmo y Recio, 1991).

\subsection{Formación y evolución de las salinas continentales del centro de Andalucía}

En los fenómenos endorreicos, así como la existencia de salinas en esta zona confluyen factores geológicos, climáticos e hídricos cuya acción conjunta permitirá la formación y conservación posterior de estos lugares.

\subsubsection{Los factores climáticos e hidrológicos}

La irregularidad y escasez de precipitaciones (raramente superan los $600 \mathrm{l} / \mathrm{m}^{2}$ ) son factores que inciden directamente en la dinámica de estas salinas. Y al clima hay que añadirle el factor hidrológico, por cuanto las aguas subterráneas, allí donde vierten a estos humedales culturales, son una fuente de alimentación fundamental (Pita, 2003).

Pero el clima del centro de Andalucía no es diferenciado respecto a su entorno (Pita, 2003), y del mismo modo tampoco es una peculiaridad subcomarcal la existencia de acuíferos subterráneos que, de una manera u otra, acaban formando surgencias, fuentes y manantiales. Por tanto, dentro de la extrema complejidad de factores que intervienen en la formación de salinas continentales, en nuestro caso quizá el factor geológico sea la clave para entender y comprender este fenómeno (Dantín, 1940).

En el estudio de las bases científicas aplicadas a nuestro estudio, resulta de especial interés abordar la relación del clima con la creación de itinerarios turísticos y educativos en las salinas continentales del Guadalquivir, ya que este factor adquiere relevancia (Gómez Martín, 2005).

Como se ha introducido antes, la posibilidad de desarrollar determinados itinerarios turísticos relacionados con la actividad salinera continental en la cuenca del Guadalquivir está muy influenciada por la aptitud climática, ya que la propia naturaleza de las salinas puede exigir condiciones climáticas y ambientales muy diferentes a otros espacios turísticos. Por ejemplo, el turismo de nieve exige una determinada aptitud invernal o el turismo de playa, una determinada aptitud climática estival (Vera, López Palomeque, Marchena, \& Clavé, 2011). Este asunto, precisamente, ha inducido la realización de estudios orientados a la sistematización de la relación clima-turismo (Figura 3), que han permitido elaborar diversos índices y formular numerosos modelos (Gómez Martín, 2005).

No obstante, desde nuestro punto de vista, queremos destacar que en el ámbito geográfico de estudio es relevante la consideración de un componente estructural del clima: el ritmo estacional, que influye en la temporalización de la actividad salinera y, en consecuencia, del turismo o de la actividad didáctica de campo. Este aspecto será desarrollado con profundidad más adelante. 


\section{Figura 3 \\ CONDICIONES PARA EL CONFORT HUMANO: TEMPERATURA Y HUMEDAD RELATIVA}

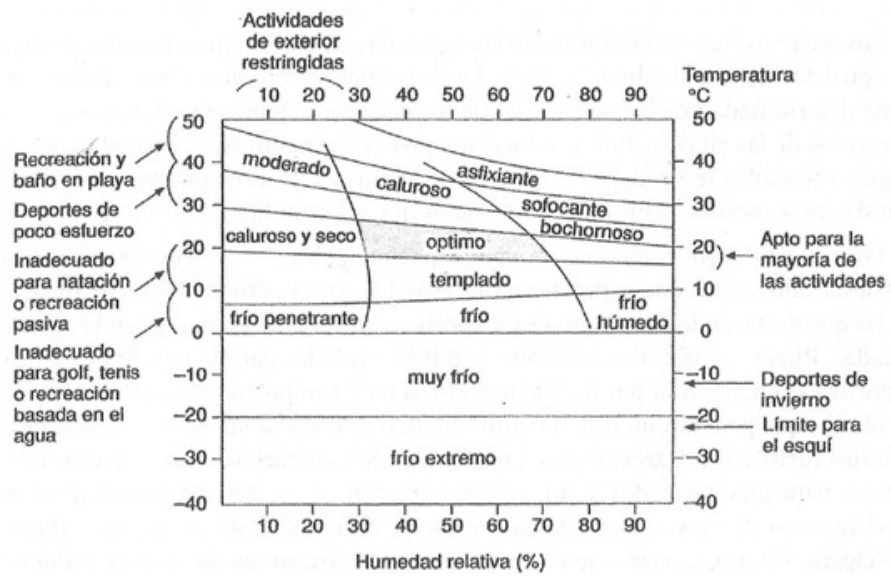

Fuente: Terjung (1966); citado por Burton (1991)

\subsubsection{El factor geológico y litológico. La importancia del Triásico}

En lo que se refiere a la geología, en los objetos de estudio predominan los materiales de la Era Terciaria, especialmente miocenos, que con frecuencia reposan directamente sobre materiales de la Era Secundaria, sin que sean apreciables los otros paquetes sedimentarios terciarios (Paleoceno, Eoceno y Oligoceno); incluso la serie estratigráfica se ve sensiblemente simplificada por la ausencia de los materiales del Jurásico y Cretácico, de manera que el Mioceno descansa con frecuencia directamente sobre el Triásico. Litológicamente predominan margas y arcillas del Trías en la base, que soportan las areniscas muy calizas de los niveles miocenos.

Nos interesa del Trías su impermeabilidad, pues frente a la facilidad de circulación subálvea que ofrecen los materiales calizo-aresniscosos terciarios, el Triásico se presenta como un estrato completamente impermeable ante el cual las aguas de infiltración, o bien son expulsadas al exterior, o bien provocan encharcamientos cuando no llega a conformarse una línea de escorrentía eficaz. Por otra parte, estos mismos materiales triásicos colaboran a la formación de cuencas cerradas de carácter endorreico, pues su disolución progresiva ha sido factor decisivo en la formación de las salinas (Roldán y García-Cortés, 1988; Quesada, 1996).

En definitiva, la zona central de Andalucía y, en especial, la incluida en las unidades de olistostroma integra situaciones adecuadas para la formación de salinas de interior: áreas cóncavas, existencia de manantiales naturales, materiales salinos subterráneos y en superficie, etc. Al no tener un drenaje fácil existen zonas donde se propicia un endorreísmo y encharcamientos más o menos prolongados que también ayudan a la existencia de estos aprovechamientos ancestrales (Rodríguez Aguilera, 1998). 
Figura 4

DEPÓSITO DE MASA DE FLUJO (“DEBRIS FLOW") CON MANTOS DE DESLIZAMIENTO. DEFORMACIÓN RELACIONADA CON LA DEPRESIÓN Y LA DEFORMACIÓN DE LA BASE (BAENA, CÓRDOBA)

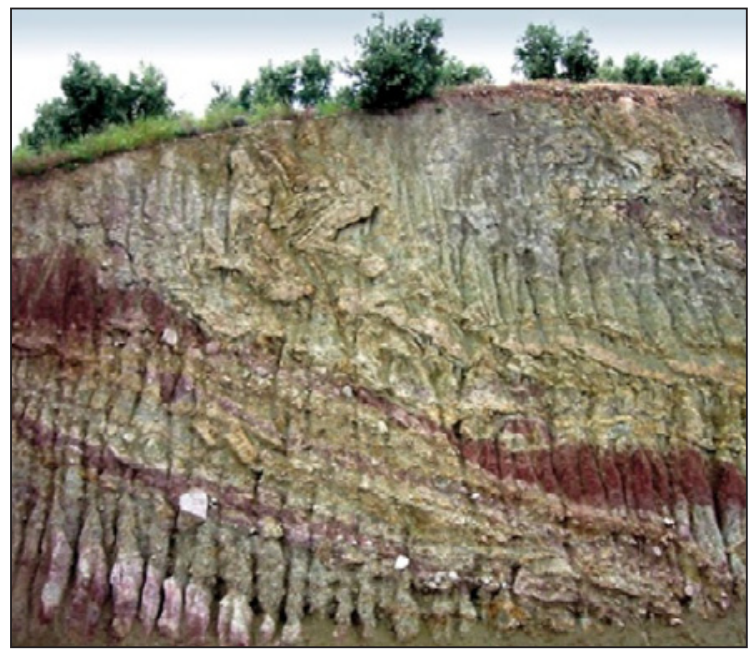

Autor: R. Vega-Pozuelo.

En la práctica totalidad de los casos, las salinas continentales del centro de Andalucía se asientan sobre espacios en los que, como resultado de la escasa potencia (o inexistencia) de algunos de los pisos del Mioceno e incluso de los materiales del Jurásico y Cretácico, el Trías se encuentra muy "cercano" (Ríos, 1963).

Figura 5

ANTIGUAS SALINAS DE LA DEHESA DEL CARRIL (LUCENA, CÓRDOBA) Y SU CUENCA VERTIENTE: OLIVOS DE NUEVA PLANTACIÓN, SOBRE MATERIALES TRIÁSICOS
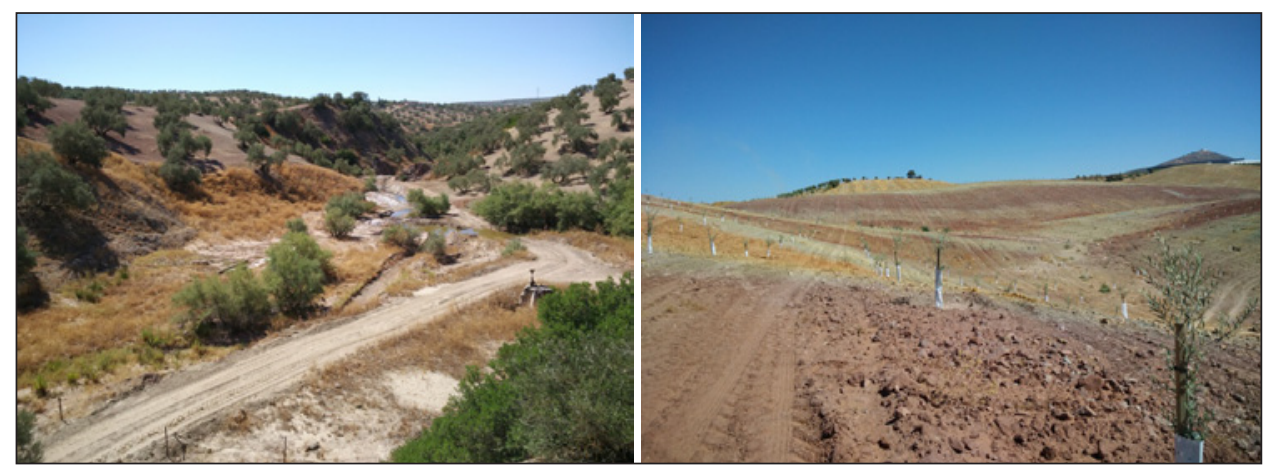

Autor: R. Vega-Pozuelo. 06/08/2016 
Según Calvo (1910), el 90\% de las fuentes saladas conocidas en España manan en terrenos del Triásico, y las demás, en terrenos del Terciario.

\section{LA SAL EN EL CENTRO DE ANDALUCÍA}

La sal común o halita $(\mathrm{NaCl})$ es un mineral muy especial e indispensable para la propia civilización y la vida en sí misma, como componente de la alimentación (Calvo, 2006).

La búsqueda de la sal por el hombre se remonta a los tiempos más remotos. Se han podido constatar restos de explotaciones salineras por evaporación, con más de 600.000 años en la Garganta de Olduvai (Tanzania) (Lekey, 1994).

En nuestro país existen indicios prehistóricos de explotación salinera de interior en numerosas localidades: Puebla del Río (Sevilla), Villafáfila (Valladolid), Ciempozuelos (Madrid), etc. Así, se conoce la presencia de salinas en nuestro entorno desde principios del siglo V a. C. tal y como es el caso de las salazones de Gadir (Cádiz). Según las fuentes de la época, la sal producida aquí era un importante objeto de comercio desde su fundación como colonia fenicia (Morere, 1991). Probablemente en esta época se obtenía ya en salinas por evaporación solar, habiéndose abandonado ya el primitivo sistema de evaporación por medio del calor del fuego, mucho menos productivo y rentable (Quesada, 1996).

En la época romana, la fabricación del "garum" obligaba a disponer de salinas en el interior, para completar la alta demanda existente. Plinio menciona las explotaciones de minas de sal en varias zonas de Iberia, y la palabra local "muria", con la que se designaba a las soluciones acuosas concentradas de las que se extraía sal en salinas (Calvo, 2006). Las salinas se consideraban propiedad del Estado romano, al igual que las minas metálicas y, también como ellas, solían explotarse por arrendamiento de compañías de "publicani". No obstante, algunas salinas de esta zona se mantuvieron en manos de sus dueños anteriores a la conquista, fueran particulares o ciudades, que debían pagar en este caso una renta fija al Estado (Madoz, 1845). La importancia de la sal en época romana es evidente si se observa que se encuentra en el origen de la palabra "salario".

En la época musulmana, la explotación de las salinas continentales se llevó a cabo con menor intensidad que la que se produciría posteriormente en la época cristiana, de tal forma que con el avance de la Reconquista se pusieron en actividad muchas pequeñas salinas en la zona de estudio (Fornell Muñoz, 2015). Según Rodríguez Aguilera (1998), la civilización cristiana era mayor consumidora de sal debido a su utilización en derivados salados y curados del cerdo, así como a las propias restricciones alimentarias del catolicismo de la época -el cual prohibía comer carne en más de un tercio de los días del año-. Eso hizo que se popularizara el consumo de pescado en salazón.

A la vista de la bibliografía consultada, podría decirse que el entramado de pequeñas salinas existentes en el centro de Andalucía procede del modelo de explotación seguido a principios de la Edad Media. Sin embargo, en el Reino de Castilla -al contrario que en el centro de Andalucía, donde se seguía bajo control político y militar andalusí- durante los siglos X y XI se produjo un proceso de concentración y la propiedad de las salinas pasó desde los primitivos explotadores a los nobles y a la Iglesia. La Corona de Castilla gravó la sal con un impuesto especial, la "albara", que se cobraba en las propias salinas o en los depósitos 
de sal por funcionarios, los "albareros", que contaban con capacidad para efectuar registros y para incautarse la sal que no tuviera el correspondiente marchamo (López Castillo, 1984).

En el año 1313, en las cortes de Palencia, Sancho IV dispuso la prohibición de establecer alfolíes particulares y de sacar del Reino la "sal de conpasso", bajo pena de muerte, y en las Cortes de Burgos de 1315 se fijaron las zonas de venta de cada salina (López Castillo, 1984). Sin embargo, la pieza fundamental en el ordenamiento jurídico sobre la sal tuvo lugar el 28 de abril de 1338 (transcrito por López Castillo, 1984 y citado por Calvo, 2006), cuando el rey Alfonso XI estableció de forma definitiva el monopolio real de la sal, al obligar a todas las salinas a vendérsela a la Corona, depositándola en los alfolíes reales y prohibiendo el comercio entre particulares.

Este régimen tuvo una nueva vuelta de tuerca durante el reinado de los Reyes Católicos, quienes reorganizaron de nuevo el monopolio de la sal mediante Pragmática de 17 de marzo de 1484, estableciendo duras penas para los contrabandistas, que podían llegar incluso a la pena de muerte por saetas (Hinojosa, 1993). Durante el siglo siguiente las salinas del centro de Andalucía se explotaron fundamentalmente mediante arriendos y Felipe II, por una ley de 10 de agosto de 1564, eliminó las adscripciones territoriales de los pueblos a determinadas salinas y alfolíes, que tantos problemas causaban, de modo que cada persona podía comprar la sal donde le conviniera, siempre que fuera en un establecimiento oficial. Como en el centro de Andalucía existía algunas salinas que no eran propiedad de la Corona de Castilla, estaba prohibido el transporte de sal andaluza de las salinas particulares a otras partes de España (Ayerbe, 1981).

En el año 1631 se procedió al estanco de la sal, encargando su gestión al Consejo de la Sal, formado por ocho consejeros, uno por cada circunscripción, que entendía de todas las causas relacionadas con el monopolio. Además, se creó un impuesto especial, el "crecimiento de la sal", por el que este producto pasaba a venderse a 32 reales por fanega (Calvo, 2006). Durante el siglo XVIII se añadieron al precio de la sal, ya muy recargado en comparación con su precio de coste, otros impuestos, que generalmente pretendieron ser de carácter temporal, para cubrir necesidades concretas y urgentes (López Castillo, 1984). A finales de este siglo, la sal pasó a desempeñar un papel importante en la naciente industria química, especialmente en la fabricación de sosa, gracias al método Leblanc que permitía obtenerla a partir del sulfato sódico natural o producido por el tratamiento de la sal con ácido sulfúrico (Rodríguez \& Rodríguez, 1999). Así, a principios del siglo XIX se produjeron intentos de liberalizar la producción y el comercio de la sal: uno por las Cortes de Cádiz, en 1813, y otro en 1821 durante el llamado "trienio liberal”, pero ninguno prosperó. En la década de 1830 la Renta de la Sal se arrendó y, en 1850 el Estado poseía un total de 69 salinas (Yegros, 1852). La ley del desestanco de la sal de 16 de junio de 1869 declaró, a partir del 1 de enero de 1870, libre fabricación y venta de la sal, estableciendo además la venta mediante subasta de las salinas pertenecientes al Estado (Calvo, 2006). El desestanco de la sal produjo también la construcción de multitud de pequeñas salinas en el centro de Andalucía, atendiendo a una simple economía de subsistencia y produciendo unas cuantas toneladas de sal al año, e incluso comercializándola mediante el trueque en vez de mediante venta. Estos sistemas se mantuvieron hasta finales de la década de 1950, cuando la mejora de las condiciones económicas y sociales acabaron con la mayoría de las pequeñas salinas continentales (Quesada, 1996). 


\section{LAS SALINAS EN EL CENTRO DE ANDALUCÍA}

La sal, históricamente, se ha medido de una forma análoga a los cereales, en volumen, no en peso (Calvo, 2006). La unidad habitual en España, hasta finales del siglo XIX, era la fanega, que en el caso de la Castellana equivale a 55,5 litros (Naranjo Ramírez, 1998). En consecuencia, el peso dependía del tamaño del grano y de la humedad que contenía la sal. "En el porte de Ávila", cada fanega de sal equivalía a 112 libras castellanas (Aldana, 1850 ), es decir, $51,56 \mathrm{~kg}$, aunque posiblemente tiene otras equivalencias según la zona. La unidad de medida empleada en las salinas de Cádiz hasta principios del siglo XX, para la carga de los barcos, era el "lastre", como unidad de peso y que equivalía a 2,25 toneladas.

En lo que se refiere a la concentración de las salmueras de las salinas y los manantiales, la forma de expresarla ha sido siempre de manera indirecta, por densidad, en grados Baumé, La equivalencia exacta entre densidad y contenido de sal no es evidente, puede considerarse que los grados Baumé se corresponden con cierta aproximación con el contenido de sal en porcentaje (CSIC, 2016).

Como se ha expuesto anteriormente, la sal es peculiar y también lo es por su forma de presentarse, ya que puede estar disuelta en agua con concentraciones de hasta $360 \mathrm{gr} / 1$.

España es uno de los países con mayor número de explotaciones salineras continentales, considerando la explotación de minas, disuelta en agua de pozo y manantiales salinos, de las que existen ejemplos importantes como se verá. Así pues, «Río Salado» es un nombre que llevan al menos once ríos en España y «arroyo Salado», al menos treinta (Recuero, 1993).

En las explotaciones salineras continentales del centro de Andalucía el agua es sometida primero a un proceso de concentración en balsas cuyo nombre, "cocederos" o "calentadores", guarda relación más con la temperatura que con la concentración, aunque sea esta última la que aumenta. Cuando se ha alcanzado una concentración próxima al punto de saturación del cloruro sódico (35,9 g por $100 \mathrm{ml}$ de agua), la salmuera pasa a las balsas de cristalización, que reciben también distintos nombres "piletas", "cristalizadores", "albercas", etc. (Quesada, 1996). La relación de superficie dedicada a balsas de un tipo o de otro depende de la concentración inicial del agua salada -en algunos casos hemos llegado a medir concentraciones de 22 grados en la escala de Baumé en el manantial (por ejemplo, salinas de Vadofresno, en Baena)-. Así, en algunos manantiales como el mencionado, el agua surge ya casi saturada en sal y el agua se acumula simplemente en depósitos hasta su paso a la etapa de cristalización.

Al sobrepasarse el punto de saturación de la sal, ésta precipita inicialmente formando una capa de micro cristales flotando sobre el agua. Si se recoge esta capa, se obtiene un producto que se conoce en nuestra zona como "flor de sal". Al crecer estos cristales, precipitan, y en el fondo continúan su crecimiento formando una costra policristalina más o menos dura según las condiciones de fabricación. En las soluciones saturadas de sal, como las que se encuentran en las salinas continentales, se produce la formación de cristales en tolva, al producirse una sobresaturación elevada y un crecimiento rápido, que hace que crezca a mayor velocidad la zona de las aristas del cubo que la parte central de las caras. Esto da lugar a la formación de crecimientos en "tolva", con cubos con las caras escalonadas hacia una depresión central, o incluso a tolvas propiamente, con aspecto de 
una pirámide escalonada, hueca, de base cuadrada (Calvo, 2006). Se forman con mucha frecuencia grupos espectaculares de cristales en torno a juncos o a briznas de hierba sumergidas en las balsas de cristalización de las salinas o en charcas naturales.

\section{Figura 6 \\ CROQUIS DE UNA “SALINILLA” COMO LA DE MONTEJÍCAR (GRANADA)}

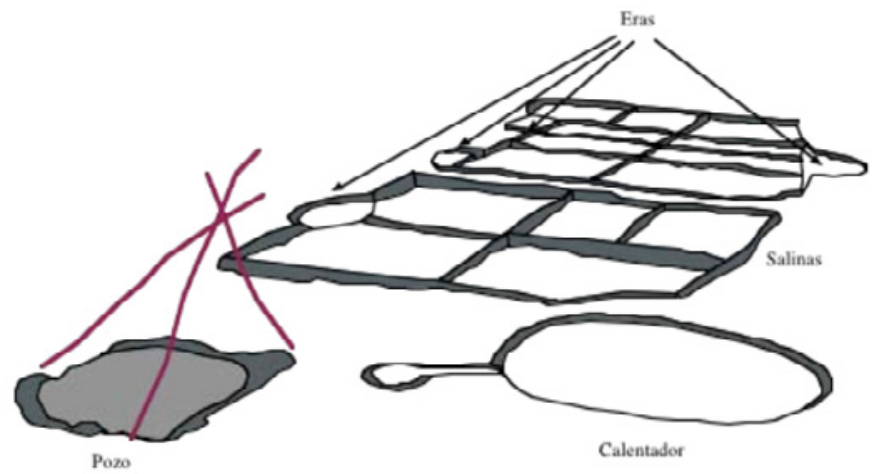

Fuente: Malpica Cuello (2005).

En nuestra área de estudio existe una gran cantidad de indicios de halita que, aunque algunos no dan lugar a yacimientos visibles, producen la salinización de las aguas subterráneas o superficiales en muchos lugares. Al menos 11 arroyos de nuestra zona de estudio tienen el nombre de Salado, por la presencia de sal disuelta en ellos. Esta abundancia de agua salada ha hecho que hasta tiempos recientes se hayan explotado en este territorio bastantes salinas. A finales de la década de 1960 en nuestra zona de estudio estaban en funcionamiento 9 salinas (Ríos, 1963). Todas ellas, con excepción de las salinas de Duernas, eran de tamaño muy pequeño, y cada una de ellas producía unas decenas o, las más grandes, unos cientos de toneladas de sal al año. En la Figura 7 se puede observar la localización de las salinas (activas o abandonadas) del centro de Andalucía, objeto de estudios más amplios por parte de los autores.

\section{LA SALINA CONTINENTAL COMO RECURSO PATRIMONIAL, GEONATU- RAL Y GEOCULTURAL}

No es difícil descubrir que las salinas continentales comprenden diversos elementos que constituyen recursos patrimoniales y, por ende, turísticos y/o didácticos y pueden actuar como factores de localización de la actuación que detallamos en nuestra investigación. El olvido de estas salinas continentales, especialmente en el territorio campiñés de clara vocación agraria, contrasta en los últimos años con cierta recuperación y difusión turística de las salinas litorales en provincias como Cádiz o Almería. Prueba de esto, por ejemplo, es la celebración en 2015, en Cádiz, de la I Feria de las Salinas. 


\section{Figura 7 \\ MAPA DE LAS SALINAS (ACTIVAS E INACTIVAS) DEL CENTRO DE ANDALUCÍA}

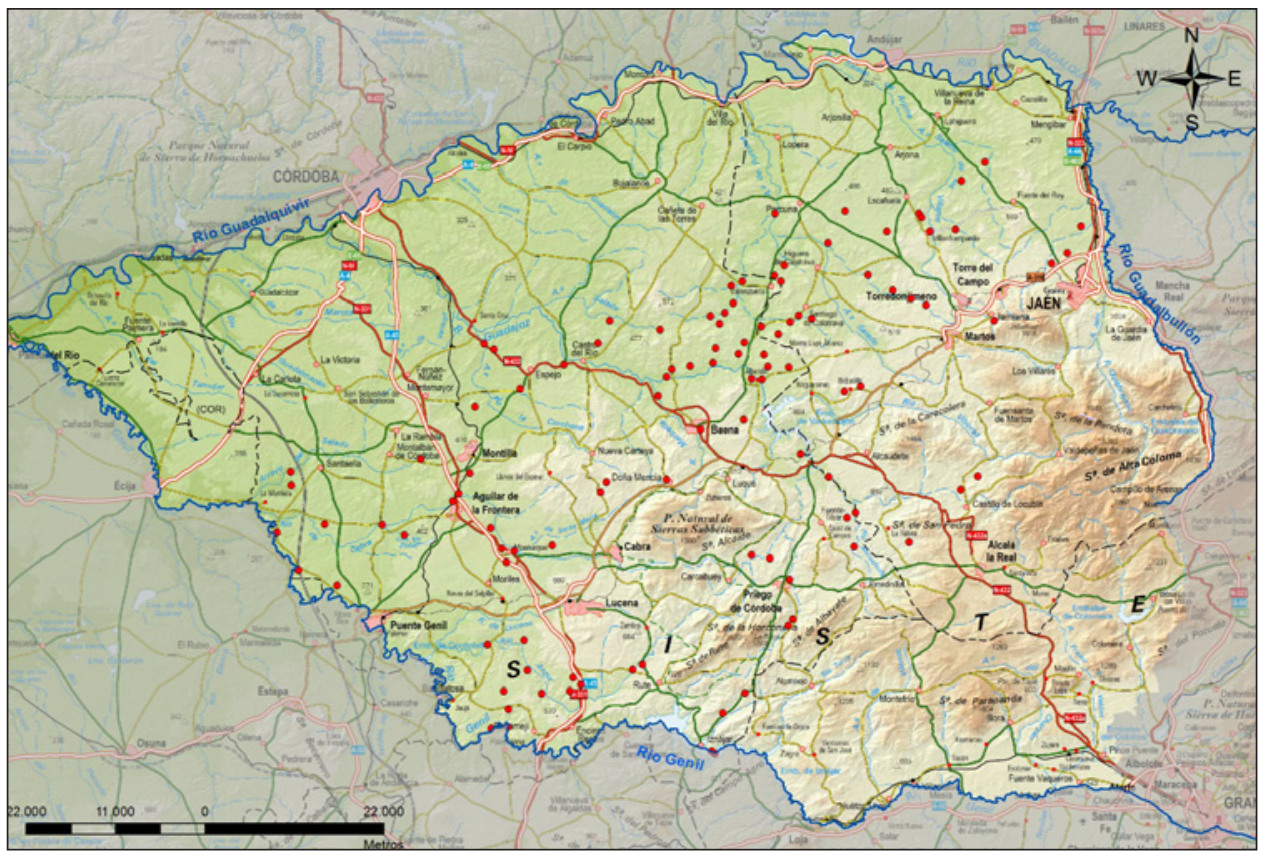

Fuente: Base cartográfica del Instituto Geográfico Nacional. Elaboración propia.

Las salinas de nuestro entorno representan (o representaban, especialmente cuando se encontraban en funcionamiento) además un elemento paisajístico de primera magnitud, con el especial reticulado de las balsas, en algunos casos de diferentes colores. El color rojizo de las balsas con mayor concentración de sal se debe, sobre todo, a la presencia de un alga microscópica: Dunaliella salina, y de bacterias halófilas.

Uno de los intereses y debates que lo anterior despierta en la sociedad actual lo hace, entre otras cosas, porque el patrimonio ha sido tradicionalmente un concepto muy sesgado hacia los valores que se denominan histórico-artísticos. En realidad, abarca mucho más que todo eso y no se debe excluir de lo patrimonial a lo derivado de la industria, más allá de los dos últimos siglos (Casanelles Rahola, 1998).

El patrimonio salinero podría adquirir, incluso, la categoría de emblemático y es considerado parte de la historia en los lugares en los que su presencia ha sido determinante para la forma de vida y trabajo de sus habitantes, la bonanza económica, la técnica o la riqueza material de la población (Álvarez Areces, 2001). Por ello, al amparo de los objetivos propuestos en la presente investigación, hay que tener en cuenta que la mayor parte de las veces, la demanda valora y percibe el conjunto de los componentes a través de una imagen global, una imagen que con frecuencia es una imagen manipulada o sesgada del paisaje natural (Callizo, 1991). 
En este sentido, responden a la acepción de patrimonio cultural e industrial. Según Pardo (2008), los elementos industriales de una salina continental no deben ser discutidos como patrimoniales; son el testimonio de épocas pasadas trascendentales para la historia de la humanidad, sólo comparable por sus efectos y avances a la revolución neolítica, así como de cultura material. Y, si se consideran las tipologías tradicionales, en concreto según la naturaleza de los recursos, se trata de recursos litom (restos arqueológicos...) y de recursos antropom (artesanía, tradiciones...).

Todo lo anterior explica que las salinas continentales del área estudiada presenten una localización de carácter puntual, y ello influirá en la distribución -factor de localizaciónde los itinerarios que se propongan.

A la vista de lo anterior, podemos concluir que el patrimonio salinero del centro de Andalucía queda implícitamente incluido como integración de paisajes con valores culturales y edificios concretos que abordan la relación del desarrollo económico y social de los individuos y las comunidades con naturaleza y el medio ambiente.

\section{Figura 8 \\ SALINAS DEL PUENTE, TAMBIÉN LLAMADAS “DE VALDETOCINOS”, CON ALBENDÍN AL FONDO (BAENA)}

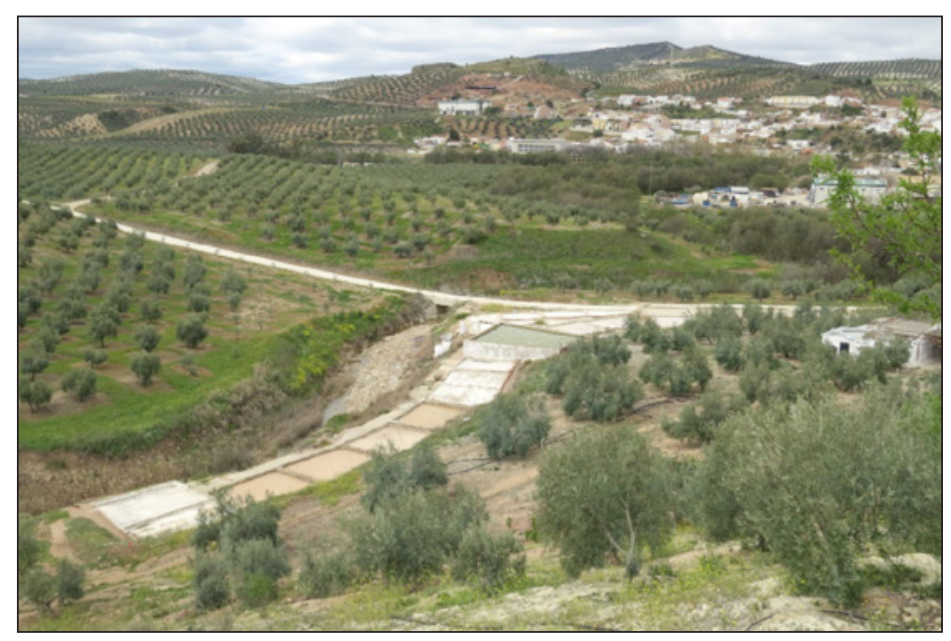

Autor: Rafael Vega-Pozuelo. 17/07/2016.

En este sentido, hemos de destacar los valores de la Carta Europea de Patrimonio, la cual hace hincapié en la idea de rehabilitación y conservación integral de los monumentos (EUROPARC, 2017). Se apuesta, además, por una conservación del patrimonio que se convierta en objetivo principal de la planificación urbana y de la ordenación del territorio.

Por ello, cabe resaltar que los itinerarios turísticos y educativos relacionados con las salinas continentales, en nuestra zona de estudio pueden suponer, además, un producto de consumo cultural que, como otros bienes, en principio superfluos, permiten adquirir una manera de conocimiento, poner de manifiesto un determinado gusto o demostrar la 
posesión de un determinado "capital cultural" (Urry, 1990). De ahí que, paralelamente a la producción de espacio, deba hablarse de la producción de imagen asociada a él (Vera, López Palomeque, Marchena, \& Clavé, 2011).

Desde este punto de vista, la zona geográfica que nos ocupa, como espacio turístico y educativo, no sólo es un espacio real y objetivo, sino que es también un espacio representado (Dewailly \& Flament, 1993).

Un paso esencial en esta línea es la necesidad de crear conciencia del paisaje, sensibilidad paisajística (Nogué, 2008). En este sentido, una estrategia paisajística de la zona de estudio podría ayudar a convertir este territorio en el motor para impulsar el turismo y la actividad didáctica y aumentar su calidad (Vilanova \& Pie, 2006).

En nuestra zona de estudio resulta especialmente relevante la gestión del paisaje derivada del mantenimiento de los sistemas agrarios tradicionales. De hecho, la extensificación y el mantenimiento de los sistemas tradicionales de uso de la tierra tienen importantes posibilidades turísticas y educativas siempre que sea bien aprovechada y reconducida al diseño de un entorno más racional (González Bernáldez, 1992).

Así pues, las motivaciones para considerar las salinas como recurso turístico o educativo son diversas: búsqueda del significado histórico y arqueológico de los lugares de antigua producción, conocimiento de un medio extraño y totalmente distinto al que existe en los medios urbanos, interés por comprender algunas técnicas en desuso, profundización nostálgica en una época y unas formas de vida ya perdidas... Este turismo o esta incursión didáctica conecta al espectador con un pasado a través de la visita a unos vestigios, museos e itinerarios (Barrado, 2011). Aparece así una nueva geografía del turismo preocupada por el simbolismo de los monumentos industriales y los territorios de la industria, en definitiva, por un patrimonio cultural de indudable interés como atractivo turístico y educativo (Pardo Abad, 2008).

\section{Figura 9 \\ SINGULARIDAD DEL ESPACIO DE DESTINO TURÍSTICO}
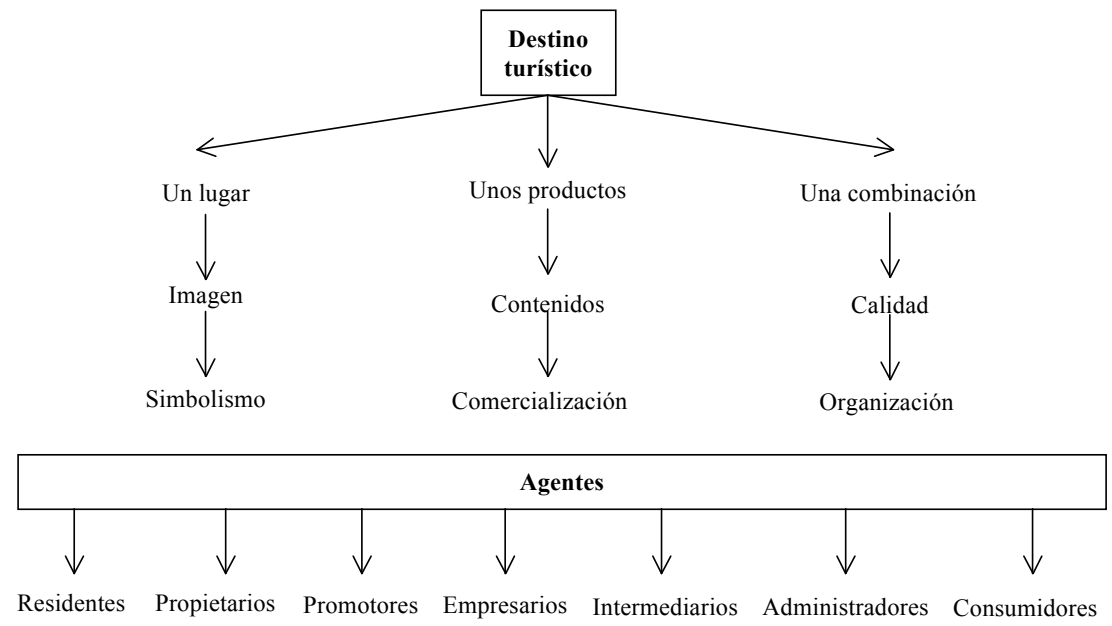

Fuente: Vera et al. (1996). 


\section{PROPUESTAS METODOLÓGICAS}

$\mathrm{Al}$ abordar las bases de la actividad turística, para la consecución de nuestros objetivos, nos resulta necesario entrar en la cuestión más general de los factores de localización de las actividades económicas, si bien conviene hacer una importante aclaración: el turismo no solo es una actividad económica, sino que es una actividad de carácter socioespacial que induce o genera diversas actividades económicas (Vera, López Palomeque, Marchena, \& Clavé, 2011). Hay que acudir, por tanto, a la conceptualización de los factores generales de localización de los hechos económicos y del turismo en particular (Figura 9), por ser este un fenómeno diverso y complejo, cuyo carácter espacial le confiere unas pautas de localización singulares (Vela-Ruiz, Aravena, \& Torres, 2013).

Para nuestros propósitos, resulta muy relevante abordar la necesidad más que demostrada de la ordenación del territorio para integrar de manera organizada y racional el espacio y la puesta en práctica de itinerarios turísticos y educativos y su papel clave para la optimización de los beneficios de su puesta en marcha.

Precisamente el manejo territorial de los flujos y de la actividad turística es objeto de estudio por parte de autores como Decoutere y otros (1996), López, Marchena, y Anton (1997) y Tuñón (1997). Partimos de la convicción de que el territorio y los humedales existentes en la zona de estudio poseen atractivos singulares y por tanto son, en sí mismos, unos recursos turísticos. Así pues, la producción turística de esta zona y su configuración podrían convertirla en un producto en sí mismo, como destino de los flujos turísticos y educacionales (Vera, López, Marchena, \& Anton, 1997) siempre y cuando se contemple una metodología adecuada en esta planificación (Figura 10).

\section{Figura 10 \\ SÍNTESIS DE LOS ASPECTOS METODOLÓGICOS ESENCIALES DE LA PLANIFICACIÓN ESTRATÉGICA}

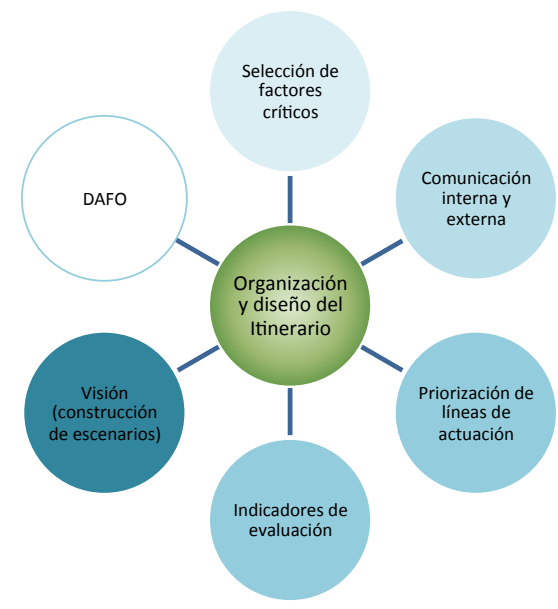

Fuente: Elaboración propia, a partir de Vera et al. (2011). 
Más allá de una simple discusión sobre la implantación y distribución de usos y sistemas que facilitan la integración funcional de un territorio, es un instrumento fundamental para evitar problemas territoriales, lograr el apoyo social e institucional y asentar principios de cooperación, cohesión y equilibrio territorial (Antón Clavé \& González Reverté, 2005).

Estas bases suponen un conjunto de instrumentos, técnicas y medidas de apoyo para los itinerarios que se puedan establecer en el centro de Andalucía.

Tabla 1

\section{INSTRUMENTOS, TÉCNICAS Y MEDIDAS DE APOYO PARA LA PROPUESTA DE ITINERARIOS TURÍSTICOS Y EDUCATIVOS DE CALIDAD SOBRE EL PATRIMONIO SALINERO}

\section{Instrumentos de ordenación, planificación territorial y planeamiento urbanístico}

- Planes y Directrices de Ordenación Territorial de ámbito regional y subregional

- Protección de espacios naturales (PORN, PRUG, etc.)

- Planes Generales de Ordenación Urbanística

- Planes Especiales de Protección de centros históricos y conjuntos monumentales

- Agendas 21 locales y provinciales

\section{Instrumentos y técnicas de gestión medioambiental}

- Evaluación de impacto ambiental

- Identificación de la capacidad de carga

- Sistemas de Gestión ambiental

- Auditorías ambientales

- Ordenanzas ambientales

- Gestión del paisaje

- Programas de vigilancia ambiental

\begin{tabular}{ll}
\hline \multicolumn{1}{c}{ Regulación de empresas y actividades turísticas y educativas } \\
\hline- & Incorporación de los principios de sostenibilidad en la legislación sectorial \\
- & Autorregulación de empresas y centros escolares o de investigación \\
- & Implantación de tasas e impuestos a empresas o directamente a la demanda \\
- & Inspección y control de las actividades turísticas y educativas \\
\hline & \multicolumn{1}{c}{ Gestión de los visitantes a las salinas continentales } \\
\hline- & Planes de Gestión \\
- & Límites del cambio aceptable \\
- & Gestión del impacto de visitantes \\
- & Experiencia del visitante y protección de los recursos \\
\hline & Técnicas basadas en la valoración económica de las salinas continentales \\
\hline- & Análisis coste-beneficio \\
- & Método de precios hedónicos \\
- & Método del coste de viaje \\
- & Método de valoración contingente \\
\hline & Medidas de apoyo \\
\hline & Información para la gestión sostenible de los itinerarios turísticos y educativos: \\
& o Creación de un sistema de indicadores de sostenibilidad \\
\hline & o Sistemas de información ambiental y de información \\
\hline
\end{tabular}


- Políticas de incentivo:

o Incorporación de criterios ambientales en los programas de ayudas y subvenciones

o Asistencia técnica

o Incentivos a buenas prácticas ambientales

- Sensibilización:

o Realización de campañas de sensibilización para residentes y visitantes

o Difusión de la información

o Difusión de buenas prácticas

o Códigos de conducta para empresas, centros y visitantes

o Fomento de canales de participación social

- Comunicación y promoción:

o Actuaciones de comunicación y promoción selectivas

o Incorporación de los principios de la sostenibilidad en el mensaje y soportes de comunicación

o "Demarketing" (desincentivar la frecuentación de áreas saturadas o especialmente frágiles)

Fuente: elaboración propia, a partir de Ivars Baidal (2003), citado por Vera et al. (2011).

Los criterios de valoración del patrimonio salinero son comunes a los del patrimonio industrial y, a su vez, a los del patrimonio arquitectónico en general (Pardo Abad, 2008), sin olvidar que tal patrimonio cuenta con una notable presencia de variables naturales y eco-culturales. A continuación, se exponen las características de los más importantes:

I. Valor histórico. En este valor se incluyen aspectos como la antigüedad de la salina, su posible carácter de hito histórico en un lugar concreto o la contribución al proceso general de desarrollo regional. También se puede tomar en consideración en este apartado la importancia económica o laboral de la salina y el papel que desempeñó dentro del sector.

II. Valor arquitectónico asociado a la salina. Las consideraciones ahora predominantes son la originalidad o representatividad tipológica o de técnicas y materiales constructivos, además del valor estético formal, la autoría de un elemento construido o su condición de hito arquitectónico.

III. Valor de conjunto. En este caso se han de valorar las salinas que conservan su arquitectura y sus diferentes instalaciones, así como las que hubiesen contribuido a generar un poblado o colonia en su entorno, combinando lo productivo con lo residencial y asistencial. En estos casos, la salina deja de ser un simple contenedor aislado o un simple objeto de culto museístico.

IV. Valor territorial. Se da cuando la salina se incluye en un conjunto más amplio de singular valor patrimonial. Se crea, en este caso, una asociación de elementos distintos que puede ser de carácter productivo (varias salinas en un mismo río o próximas entre sí), residencial-productivo (una salina y su correspondiente colonia industrial), paisajístico-ambiental, etc.

V. Valor iconográfico. Tiene que ver con el valor simbólico del que son portadores los elementos de la salina, es decir, la capacidad de sugerir o evocar realidades 
históricas más amplias o paisajes salineros pretéritos. La conservación de eras de evaporación empedradas, por ejemplo, como elementos altivos y herencia del pasado, cumple esta función.

VI. Valor de conservación. Más que ningún otro patrimonio, el salinero requiere funcionar o ser reutilizado. El estado de conservación es un criterio importante, aunque muchas veces se identifican a las casas salinera y a los silos como ruinas, cuando sólo presentan algunos defectos leves de conservación, esto demuestra que la sociedad es muy exigente con este legado y considera que, al concluir su función productiva, no es necesario prolongar artificialmente su existencia.

VII. Valor de uso. Se da generalmente en salinas de gran tamaño. Este valor adquiere en el patrimonio salinero una importancia trascendental, casi desconocida en otros ámbitos del patrimonio cultural.

VIII. Valor turístico y didáctico. Se relaciona con la preservación y difusión cultural y ambiental de las herramientas e instalaciones, elementos especialmente amenazados por el abandono de las explotaciones. En ocasiones, estos componentes constituyen los restos más significativos de las salinas y, debidamente equipados, pueden constituir verdaderos productos turísticos/didácticos.

Tabla 2

CRITERIOS DE VALORACIÓN DEL PATRIMONIO SALINERO

\begin{tabular}{l|l}
\hline \multicolumn{1}{c|}{ Valor } & \multicolumn{1}{c}{ Indicadores principales } \\
\hline Histórico & $\begin{array}{l}\text { Antigüedad de la salina, posible hito histórico de un lugar, papel desempe- } \\
\text { ñado en el desarrollo industrial... }\end{array}$ \\
\hline Arquitectónico & $\begin{array}{l}\text { Originalidad y representatividad tipológica, técnicas y materiales emplea- } \\
\text { dos, estética formal, consideración de hito en el paisaje... }\end{array}$ \\
\hline De conjunto & Presencia de las diferentes instalaciones fabriles, poblado industrial... \\
\hline Territorial & Asociación de elementos productivos, paisajísticos, territoriales... \\
\hline Iconográfico & Simbolismo de los elementos salineros, capacidad de evocación del pasado... \\
\hline De conservación & Estado de conservación, capacidad de puesta en uso... \\
\hline De uso & $\begin{array}{l}\text { Utilización de las estructuras, posibilidad de potenciar la identidad de la } \\
\text { comunidad local... }\end{array}$ \\
\hline Turístico-educativo & $\begin{array}{l}\text { Preservación de máquinas e instalaciones para la explicación del proceso } \\
\text { productivo, un paisaje industrial, un territorio... }\end{array}$ \\
\hline
\end{tabular}

Fuente: adaptación de Pardo Abad (2008).

A todo lo anterior, hay que añadir la necesidad de tener en cuenta una serie de criterios específicos, como son:

- La cercanía a una vía principal de acceso y posibilidad de ofrecer aparcamiento a los visitantes; 
- existencia o no de iniciativas de desarrollo o conservación en la zona y potencialidad turística o educativa:

- tipología de la salina (edificios asociados, pequeñas naves, etc.), valor histórico y flexibilidad de espacios;

- posibilidad o no de incorporar nuevos edificios y enriquecer la imagen de la salina original;

- provisión de nuevos servicios, evaluación de las diferentes estrategias de promoción y desarrollo de un plan de conservación, entre otros (Piñera y Millán, 2016).

Los análisis parciales efectuados en nuestro estudio, aún todavía en curso, nos permiten realizar un diagnóstico de las debilidades, amenazas, fortalezas y oportunidades (análisis DAFO) para concretar las actuaciones más relevantes para el futuro desarrollo de itinerarios turísticos y educativos de calidad sobre el patrimonio salinero en el centro de Andalucía. Este análisis se concreta en lo dispuesto en la Tabla 3.

\section{Tabla 3}

\section{DIAGNÓSTICO DE LAS DEBILIDADES, AMENAZAS, FORTALEZAS Y OPORTUNIDADES (DAFO) PARA LA CREACIÓN DE ITINERARIOS TURÍSTICOS SOBRE EL PATRIMONIO SALINERO EN EL CENTRO DE ANDALUCÍA}

\begin{tabular}{|c|c|}
\hline Fortalezas & Debilidades \\
\hline $\begin{array}{l}\text { - Labor realizada por la comunidad científica } \\
\text { - Disponibilidad de elementos salineros de } \\
\text { gran interés para su puesta en valor } \\
\text { - Buena conservación de algunas salinas } \\
\text { (Duernas, por ejemplo) } \\
\text { - Sinergia entre naturaleza, minería, arqueolo- } \\
\text { gía, arquitectura, etc. } \\
\text { - Potencial recursos eco-cultural novedoso y } \\
\text { adecuado en el contexto del turismo alterna- } \\
\text { tivo de ámbitos eminentemente rurales. }\end{array}$ & $\begin{array}{l}\text { - Conflictos entre usos turístico-educativo y } \\
\text { propiedad privada } \\
\text { - Insuficiente puesta en valor y difusión del } \\
\text { patrimonio, tanto natural como arquitectó- } \\
\text { nico, arqueológico e industrial } \\
\text { - Mal estado de conservación o eliminación } \\
\text { del patrimonio minero en multitud de salinas } \\
\text { (Roblizas, Cuesta Paloma, La Orden...) } \\
\text { - Falta de inventarios exhaustivos con valora- } \\
\text { ción cultural o turística, así como inexisten- } \\
\text { cia de señalización, gestión, interpretación y } \\
\text { difusión de los valores eco-culturales de las } \\
\text { tradicionales salinas interiores } \\
\text { - Débil promoción del área de estudio y de sus } \\
\text { recursos como destino turístico y educativo. }\end{array}$ \\
\hline Amenazas: & Oportunidades: \\
\hline $\begin{array}{l}\text { - El deterioro de las instalaciones, que incre- } \\
\text { mentan los costes de reparación y restau- } \\
\text { ración, cuando no está significando la } \\
\text { desaparición de un importante patrimonio } \\
\text { prácticamente desconocido en el contexto } \\
\text { del paisaje rural mediterráneo andaluz, pues }\end{array}$ & $\begin{array}{l}\text { - Declaración del patrimonio salinero como } \\
\text { BIC } \\
\text { - Romanticismo y nostalgia en sectores de } \\
\text { avanzada edad } \\
\text { - Situación central del área de estudio en el } \\
\text { contexto del territorio andaluz, así como la }\end{array}$ \\
\hline
\end{tabular}




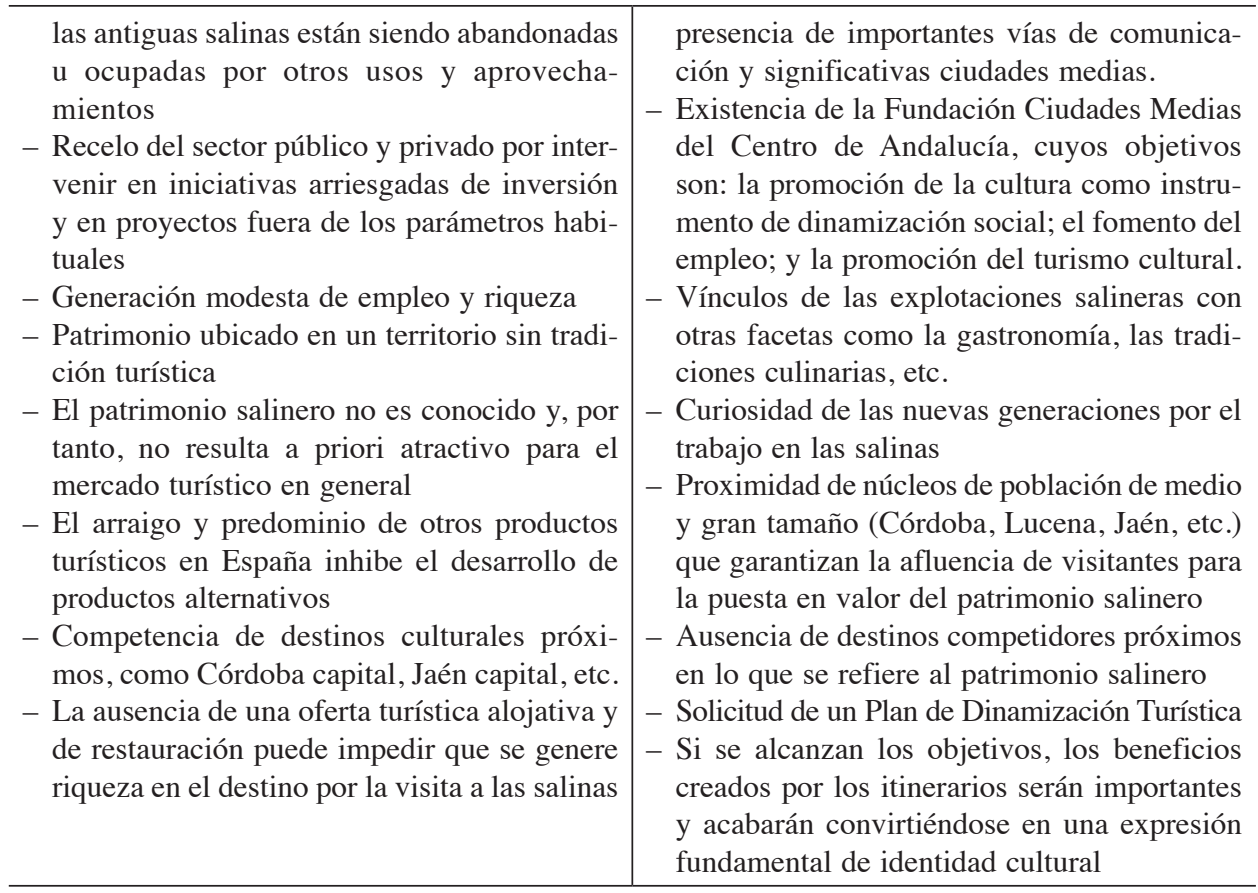

Fuente: elaboración propia.

\section{CONCLUSIONES}

Desde la perspectiva seguida en nuestra investigación, se enfatiza que una positiva inserción de itinerarios turísticos y educativos relacionados con las salinas continentales en la cuenca del Guadalquivir, entre los ríos Guadalbullón y Genil, permitirá la conservación de parte de este patrimonio territorial y facilitaría su conocimiento mediante su conversión en producto cultural, didáctico y turístico. Sin embargo, actualmente este potencial está muy lejos de ser viable, pues su desarrollo requeriría de un notable esfuerzo por parte de las instituciones que los promuevan, como de los agentes que intervengan y de los residentes a quienes afecten (Vera y Anton Clavé, 1996; Ivars, 2003).

Por ello, resulta necesario recalcar que las propuestas aquí realizadas y analizadas cuentan con las siguientes características:

- La robustez conceptual de los itinerarios turísticos-educativos y de las posibilidades que se derivan de su desarrollo.

- La definición de los niveles y tipos de impactos económicos, ambientales y sociales en el proceso de desarrollo.

- La definición de los elementos básicos de la estructura del territorio y la configuración del marco de referencia para las actuaciones de las distintas administraciones públicas concernidas, sobre la base del principio de cooperación territorial e interadministrativa. 
- La aceptación de límites para la actividad a desarrollar a fin de evitar impactos territoriales irreversibles.

Como se ha indicado en el análisis DAFO, si se cumplen estos aspectos, los beneficios creados por los itinerarios turísticos y educativos sobre el patrimonio salinero en la zona de estudio serán importantes y acabarán convirtiéndose en una expresión fundamental de identidad cultural. Entre esos beneficios destacan los siguientes (Fernández Zambón \& Ramos Schenk, 2005): consolidar la cultura productiva regional; dinamizar las economías regionales o locales; sensibilizar y concienciar de la importancia del patrimonio salinero para recuperar la identidad de los pueblos; incorporar a los grandes circuitos; preservar el patrimonio salinero y dar a conocer las condiciones de trabajo y los procesos técnico-productivos, actuales y pasados; y promover el desarrollo productivo local a partir de un plan estratégico para el patrimonio salinero y su valoración turística (Vera, López Palomeque, Marchena, \& Clavé, 2011).

Los itinerarios posibles, en función de un análisis previo de casos, titularidad, condiciones de uso, servicios necesarios de acogida, musealización, restauración e interpretación de los hitos que se incluyeran tienen la ventaja de que dan unidad a los elementos dispersos sobre el territorio: las salinas continentales y, a éstas, importancia y significado cultural dentro de un conjunto. Un itinerario permite reconstruir de manera progresiva los procesos de extracción de la sal y unas condiciones de vida propias de otras épocas que se vinculan al patrimonio salinero como valores asociados (Pardo Abad, 2008).

En cualquier caso proponemos, además de lo anterior, otras tres claves que parecen estar dentro de los límites de lo posible, entre la realidad y el deseo, en la propuesta de itinerarios turísticos y didácticos relacionados con el patrimonio salinero en el interior de Andalucía (Vera \& Anton Clavé, 1996):

- Necesidad de que se expresen las relaciones urbanísticas en la zona de estudio, incluyendo la promoción turística y la sostenibilidad adecuada de las salinas, así como la protección de éstas en el devenir urbano de los respectivos municipios afectados.

- La ordenación del paisaje y la cualificación ambiental deben ser los vértices de toda la planificación territorial de esta unidad, con una doble estrategia: 1) diseñar con criterios flexibles, sostenibles y competitivos las redes turísticas y 2) que estos diseños sean factibles para su comercialización turística, con criterios de bajo impacto y alta integración territorial y social.

- Una gestión concertada del patrimonio salinero, en su vertiente turística y didáctica, donde se produzca una doble relación entre los poderes públicos (especialmente en los ámbitos local, provincial y regional), con la sociedad receptora, que debiera conocer y participar en esta estrategia, así como con los agentes económicos y sociales que están implicados.

\section{BIBLIOGRAFÍA}

ALDANA, L. d. (1850): «Salinas de Añana», Revista Minera, n 1, pp. 103-113.

ÁLVAREZ ARECES, M. (2001): «Patrimonio industrial, identidad cultural y sostenibilidad», en Arqueología industrial, patrimonio y turismo cultural. Gijón, Incuna. Asociación de Arqueología Industria, pp. 13-31. 
ANTÓN CLAVÉ, S. y GONZÁLEZ REVERTÉ, F. (2005): «Fundamentos de planificación territorial», en Planificación territorial del turismo. Barcelona, UOC, pp. 15-60. AYERBE, M. (1981): «La industria de la sal en las salinas de Léniz y Gaviria», Boletín de la Real Sociedad Bascongada de los Amigos del País, no 37, pp. 245-269.

BARRADO, D. (2011): «Recursos territoriales y procesos geográficos: el ejemplo de los recursos turísticos», Estudios Geográficos, vol. LXXII, nº 270, pp. 35-58.

CALLIZO, J. (1991): Aproximación a la geografía del turismo. Madrid: Síntesis.

CALVO, M. (2006): Minerales y Minas de España. Vol. III - Halogenuros. Álava, Museo de Ciencias Naturales de Álava.

CALVO, S. (1910): Los minerales de España. Madrid, Junta para ampliación de estudios e investigaciones científicas.

CASANELLES RAHOLA, E. (1998): «Recuperación y uso del patrimonio industrial», Ábaco. Revista de Ciencias Sociales, $\mathrm{n}^{\circ}$ 19, pp.11-18.

CSIC. (2016): «Index of datos del Instituto de Acuicultura de Torre de la Sal». Recuperado el 29 de agosto de 2017, de http://www.iats.csic.es/datos/

DANTÍN, J. (1940): «La aridez y el endorreísmo en España. El endorreísmo bético», Estudios Geográficos, nº 1, pp. 75-117.

DECOUTERE, S., ET AL. (1996): Le management territorial. Lausana, C.E.A.T.

DEWAILLY, J. y FLAMENT, E. (1993): Géographie du tourisme et des loisirs. Paris, Sedes.

DÍAZ DEL OLMO, F. y RECIO ESPEJO, J. (1991): «Lagunas y áreas lacustres continentales de Andalucía Occidental (geomorfología, suelos y evolución cuaternaria)», Cuadernos de Información Geográfica, n 17, pp. 25-36.

EUROPARC (2017): «Portal web de la Carta Europea de Turismo Sostenible». Recuperado el 31 de agosto de 2017, de http://www.redeuroparc.org/actividades/cartaeuropea-turismo-sostenible

FERNÁNDEZ ZAMBÓN, G. y RAMOS SCHENK, A. (2005): «Patrimonio industrial y rutas turísticas culturales: algunas propuestas para Argentina», Cuadernos de Turismo, $\mathrm{n}^{\mathrm{o}} 15$, pp. 97-112.

FORNELL MUÑOZ, A. (2015): «Aproximación al uso de los humedales de la Campiña jiennense en época romana». En Qui lacus aquae stagna paludes sunt... Estudios históricos sobre humedales en la Bética. Cádiz, Seminario Agustín de Horozco de Estudios Económicos de Historia Antigua y Medieval, pp. 91-120.

GÓMEZ MARTÍN, M. (2005): «Reflexión geográfica entorno al binomio clima-turismo», Boletín de la Asociación de Geógrafos Españoles, n40, pp. 111-134.

GONZÁLEZ BERNÁLDEZ, F. (1992). «Turismo y medio ambiente», Revista Valenciana d'Estudis Autonòmics, nº13, pp. 139-166.

HINOJOSA, J. (1993): «Las salinas del mediodía alicantino a fines de la Edad Media», Investigaciones Geográficas, $\mathrm{n}^{\circ} 11$, pp. 279-292.

IVARS BAIDAL, J. (2003): «Política local y gestión de los espacios turísticos, en Santos Solla», en La Geografía y la gestión del turismo. Santiago de Compostela, Universidad de Santiago de Compostela, pp. 17-58.

IVARS, J. (2003): Planificación turística de los espacios regionales en España. Madrid, Síntesis. 
LEKEY, M. (1994): Bed III. Site JK (Juma>s Korongo). En O. Gorge, Excavation in Bed III and IV (1968-1971) and Masek Beds (pp. 15-35). Cambridge, Cambridge University Press.

LÓPEZ CASTILLO, S. (1984): «El ordenamiento jurídico del comercio de la sal y Salinas de Añana (Alava)», Anuario de Estudios Medievales, n 14, pp. 441-446.

LÓPEZ ONTIVEROS, A. (2005): «Rasgos geográficos de la Campiña de Córdoba», en Julio César y Corduba: tiempo y espacio en la campaña de Munda (49-45 A.C.). Córdoba, Universidad de Córdoba, pp. 13-65.

LÓPEZ PALOMEQUE, F. (1993): «Modalidades turísticas y tipologías de espacios turísticos», Papers de Turisme, $\mathrm{n}^{\circ} 11$, pp. 49-64.

MADOZ, P. (1845): Diccionario geográfico-estadístico-histórico de España y sus posesiones de Ultramar. Madrid, Pascual Madoz.

MALPICA CUELLO, A. (2005): «Producción y comercio de la sal marítima en la España Mediterránea en época medieval», en El Mediterráneo: la cultura del mar y la sal. Santa Pola, Consejería de Cultura, pp. 129-147.

MIECZKOWSKI, Z. (1995): Environmental issues of tourism and recreation. Laham, University Press of America.

MORERE, N. (1991): «L〉explotaition romaine du sel dans la region de Sigüenza», Gerión. Revista de Historia Antigua, no 3, pp. 223-236.

NARANJO RAMÍREZ, J. (1998): Transcripción literal del Interrogatorio General del Catastro de Ensenada correspondiente a Aguilar de la Frontera. Córdoba, Diputación Provincial de Córdoba.

NARANJO RAMÍREZ, J. (2013): «Las campiñas del Guadalquivir: claves para una interpretación geográfica», Revista de Estudios Regionales, n 96, pp. 99-134.

NOGUÉ, J. (2008): «Paisaje, territorio y sociedad civil», en Retorno al paisaje. El saber filosófico, cultural y científico del paisaje en España. Valencia, EVREN, pp. 217-242.

PARDO ABAD, C. (2008): Turismo y patrimonio industrial. Madrid, Síntesis.

PIÑERA, M. y MILLÁN, M. (2016): «Recuperación del patrimonio, turismo y desarrollo local. Un estudio de caso: la finca del Menjú», en Retos y tendencias de la Geografía Ibérica. Murcia, Universidad de Murcia, pp. 722-731.

PITA, M. (2003): «El clima de Andalucía», en Geografía de Andalucía. Barcelona, Ariel, pp. 137-174.

QUESADA, T. (1996): «Las salinas de interior de Andalucía oriental: ensayo de tipología». En Agricultura y regadío en Al-Andalus. Almería, pp. 317-333.

RECIO, J. (1989): «Endorreísmo y lagunas andaluzas», El Cuaternario en Andalucía Occidental. Sevilla, AEQUA, pp. 99-104.

RECUERO, A. (1993): «En España subsisten unas 38 salinas de manantial. Viejo oro blanco», Revista del Ministerio de Obras Públicas y Transportes, n 406, pp. 18-24.

RÍOS, J. (1963): Materiales salinos del suelo español (Vol. 64). Madrid, Instituto Geológico y Minero de España.

RODRÍGUEZ AGUILERA, Á. (1998): Las salinas de la Campiña de Jaén en la Edad Media. La Orden de Calatrava y el Concejo de Jaén. Jaén, Universidad de Jaén.

RODRÍGUEZ, J. y RODRÍGUEZ, D. (1999): «Los procedimientos clásicos de fabricación de la sosa», Ensayos: Revista de la Facultad de Educación de Albacete, $\mathrm{n}^{\circ}$ 14, pp. 293-310. 
ROLDÁN, F. y GARCÍA-CORTÉS, A. (1988): «Implicaciones de los materiales triásicos en la Depresión del Guadalquivir, Cordilleras Béticas (provincias de Córdoba y Jaén)», en II Congreso Geológico de España. Granada, Universidad de Granada, pp. 189-192. TUÑóN, J. (1997): «La gestión de los recursos económicos en el municipio turístico», en VI Simposio Internacional de Turismo. Barcelona, ESADE, p. 26.

URRY, J. (1990): The Tourist Gaze: Leisure and Travel in Contemporary Societes. London, Sage.

VELA-RUIZ, G., ARAVENA, J. y TORRES, J. (2013): «Investigación, Planificación y Estudio del Potencial Turístico del Parque Nacional Bernardo O’Higgins, Patagonia Chilena», en V Congreso Latinoamericano de Investigación Turística, Sao Paulo, pp. 159-176.

VERA, J. y ANTON CLAVÉ, S. (1996): «Métodos y técnicas para la planificación turística del territorio», en Turismo y planificación del territorio en la España de fin de siglo». Tarragona, Universitat Rovira i Virgili, pp. 6-44.

VERA, J., LÓPEZ PALOMEQUE, F., MARCHENA, M. y ANTON, S.(2011): Análisis territorial del turismo y planificación de destinos turísticos. Valencia, Tirant lo Blanc.

VERA, J., LÓPEZ, F., MARCHENA, M. y ANTON, S. (1997): Análisis territorial del turismo. Barcelona, Ariel.

VILANOVA, J. y PIE, R. (2006): «El medio y el paisaje. Los argumentos centrales del nuevo planeamiento urbanístico de la Costa Brava. La revisión del Plan General de Torroella e Montgrí, en El paisaje y la gestión del territorio», en Criterios paisajísticos en la ordenación del territorio y el urbanismo. Barcelona, Centre Ernest Lluch y Diputación de Barcelona, pp. 549-568.

YEGROS, S. (1852): «Apuntes sobre Salinas. Noticias de las salinas de España por distritos mineros», Revista Minera, $\mathrm{n}^{\circ} 3$, pp. 197-304. 
